\title{
Antonio Scurati e IL bambino che sognava la fine DEL MONDO: UNO SCRITTORE AI CONFINI TRA REALTÀ, FINZIONE E AUTOFINZIONE
}

\section{Anna Chiafele}

Riassunto: Il seguente saggio intende analizzare un romanzo di Antonio Scurati: Il bambino che sognava la fine del mondo (2009). Quest'opera, ai confini tra realtà, finzione e autofinzione, si inserisce perfettamente all'interno del dibattito sul New Realism. Scurati non ci offre solamente la rielaborazione romanzesca di due storie di presunta pedofilia, ma ci propone una spiegazione di come una macchinazione possa essere montata, a livello nazionale, grazie ai giornali, alla televisione ed a altri mezzi di comunicazione. Partendo da alcune teorie di Jean Baudrillard sul simulacro e sulla iperrealtà, vengono qui messi in risalto alcuni dei meccanismi che generano una realtà simulata, la quale risulta essere più reale del reale e trasporta l'essere umano all'interno di un costante reality show. In una realtà trasformatasi in reality show svaniscono i labili confini tra attori e spettatori, tra vittime e carnefici, tra accusatori e accusati, e tra realtà e finzione e questo crea smarrimento in una società postindustriale quale la nostra, in cui si vive attanagliati da una paura le cui origini sono difficili da identificare.

A partire dalla metà degli anni Novanta, numerosi episodi di cronaca sono diventati argomento principale nella narrativa italiana e sono stati fonte di riflessione in romanzi di scrittori quali Giuseppe Genna, Antonio Franchini, Gianfranco Bettin e Edoardo Albinati. Ciò ha spinto alcuni critici a parlare di un "ritorno alla realtà," di "faction," ovvero dell'unione tra fiction e non-fiction, tra fiction e facts. In Italia, il dibattito sul New Realism è stato sollecitato da scritti quali

\footnotetext{
${ }^{1}$ Donnarumma, Raffaele e Policastro, Gilda, a cura di. "Ritorno alla realtà? Otto interviste a narratori italiani." Allegoria 57 (2008): 9-25.

${ }^{2}$ Cfr. Donnarumma, Raffaele in Hanna Serkowska, pp. 24-25.
} 
Memorandum 1993-2008: narrativa, sguardo obliquo, ritorno al futuro ${ }^{3}$ del gruppo Wu Ming e il n. 57 della rivista Allegoria. A tutt'oggi questa vivace discussione resta aperta e non sembra ancora esaurita, soprattutto dopo la pubblicazione, nel 2012, di Manifesto del nuovo realismo di Maurizio Ferraris e della conseguente diatriba tra quest' ultimo e Gianni Vattimo. ${ }^{4}$ Come sostiene Angelo Guglielmi, gli scrittori italiani sono affascinati dal New Realism e ne subiscono la "suggestione solleticante ma insieme ne temono il rischio cui è esposto" (28). In questo gruppo di intellettuali si può inserire anche Antonio Scurati, docente prima all'Università degli Studi di Bergamo e ora ricercatore allo IULM di Milano. ${ }^{5}$ Scurati si colloca perfettamente, nel 2009, al centro della disputa tra la fine del postmoderno e il ritorno alla realtà e, con il suo romanzo Il bambino che sognava la fine del mondo, sembra forzare le barriere tra postmodernismo e nuovo realismo.

Consapevole del dibattito che si è sviluppato sul ritorno alla realtà, Scurati sostiene che nel territorio del "fictual" "la distinzione tra il finzionale (fictional) e il fattuale (factual) non è più rilevante, prima ancora di non essere possibile [...]" (Letteratura 24). In esergo al romanzo Il bambino che sognava la fine del mondo l'autore avverte il lettore che il testo:

appartiene al genere di componimenti misti di cronaca e d'invenzione. Poiché ritiene che la vocazione della letteratura sia oggi, in un tempo dominato dalla cronaca, non già quella di confondere ulteriormente i confini tra realtà e finzione, bensì quella di superarli, l'autore invita il lettore a considerare ogni singola parola di questo libro come frutto della sua immaginazione, anche e soprattutto quando si narri di fatti riferiti a personaggi e contesti che portano il nome di persone o di istituzioni realmente esistenti. (4)

\footnotetext{
${ }^{3}$ Il memorandum è apparso in rete il 12 settembre 2008 e successivamente è stato incluso in New Italian Epic. Letteratura, sguardo obliquo, ritorno al futuro, pubblicato dall'editore Einaudi. Può essere ancora scaricato al seguente indirizzo: «http://www.wumingfoundation.com/italiano/ WM1_saggio_sul_new_italian_epic.pdf». 18 aprile 2014.

${ }^{4}$ Per una ricca rassegna sul nuovo realismo si rimanda al progetto di LabOnt, presso l'Università degli Studi di Torino: «http://nuovorealismo.wordpress.com/». 18 aprile 2014.

${ }^{5}$ Nel saggio "Il romanzo sociale senza morti ammazzati” Novelli cita Saviano e Scurati tra quegli autori significativi che hanno saputo fare della loro scrittura un fenomeno della cosidetta faction (38).
} 
Nel romanzo qui discusso anche il narratore-protagonista riflette sull'ideologia del nuovo realismo che, a suo parere, si sarebbe impossessata della televisione negli ultimi venti anni e che sarebbe dilagata ovunque, in ogni ambito sociale e culturale, ed anche, per l'appunto, nella letteratura contemporanea (Il bambino 83). ${ }^{6}$ In un'intervista del 2009, l'autore si spinge persino a dichiarare "impertinente" ("Retorica") la distinzione tra reale e fittizio; guardando la televisione, gli spettatori, infatti, entrerebbero in una dimensione in cui la distinzione tra il reale e il fittizio non è più possibile. In questa sede si vuole analizzare come Scurati riesca a fornire un'acuta interpretazione della realtà trasportando i lettori ben oltre la finzione narrativa e svelandogli alcuni dei meccanismi che generano una realtà simulata, ovvero una iperrealtà, che risulta essere più reale del reale e trasporta l'essere umano all'interno di un perpetuo reality show. ${ }^{7}$ Questa discussione prende ampio spunto da alcune delle principali teorie sulla simulazione, simulacro e iperrealtà elaborate dal filosofo francese Jean Baudrillard (1929-2007). ${ }^{8}$

Prima di procedere nell'analisi, però, è opportuno sottolineare l'assidua partecipazione dello scrittore Scurati al mondo editoriale e al sistema mediatico italiano. ${ }^{9}$ Scurati ha scritto sia saggi che romanzi per Bompiani, collabora come editorialista per "La Stampa", e appare regolarmente in televisione come opinionista. L'autore ha tenuto anche una sua rubrica Lettere dal Nord all'interno del programma Rai Parla con me di Serena Dandini e Andrea Salerno. Infine, l'autore è ricercatore universitario di teorie e tecniche del linguaggio televisivo allo Iulm di Milano. Grazie a questa sua preparazione, lo scrittore conosce i trucchi e le tecniche del linguaggio televisivo e, più di chiunque altro, sembra autorizzato

\footnotetext{
${ }^{6}$ Il rapporto tra finzione, cronaca, realtà e, soprattutto, memoria viene elaborato dall'autore anche nel romanzo Il sopravvissuto (2005). A tale proposito, si veda Gabriella De Angelis in Hanna Serkowska, 393-406.

${ }^{7}$ Nel saggio "Ibridare finzione e realtà" Bruno Falcetto cita brevemente Antonio Scurati e il suo Il bambino che sognava la fine del mondo. Secondo Falcetto questo romanzo si muove verso i lettori "a partire dalla cornice del discorso di finzione" (63).

${ }^{8}$ Partire da Jean Baudrillard non è affatto una scelta casuale. In Stile e tradizione nel romanzo italiano contemporaneo anche Alberto Casadei sostiene che il pensiero di Scurati, così come viene esposto nel suo La letteratura dell'inesperienza, riesce a "sintetizzare bene riflessioni formulate da Benjamin e Heidegger, e più recentemente da [...] Baudrillard [...]” (25).

${ }^{9}$ Per Andrea Di Consoli, Antonio Scurati è "divorato dal demone del successo" (81) e identifica il "tallone d'Achille (82)" dello scrittore proprio nella sua assidua presenza pubblica che "depotenzia la sua statura letteraria" (82).
} 
ad esprimere la propria opinione su un mondo, quello mediatico, che, pur avviluppandoci, non ci appartiene. Dalle pagine de Il bambino che sognava la fine del mondo affiora fortemente la formazione culturale di Scurati e quindi, anche per questo, i giudizi qui espressi verso i media si caricano di una fortissima valenza polemica e parodica. E polemica c'è proprio stata poco dopo l'assegnazione del premio Strega nel 2009. Con questo romanzo, arriva secondo, per un solo voto, dopo Tiziano Scarpa con il suo Stabat Mater. Secondo Scurati, il vincitore Scarpa avrebbe insinuato il sospetto che i numerosi suoi successi, nel campo giornalistico, accademico, editoriale e culturale, fossero stati conseguiti grazie a favori da lui ricevuti da parte di persone influenti. L'autore ha ribattuto a Scarpa dichiarando:

Io sono arrivato ovunque da outsider, ma una volta entrato nel sistema non ho finto di essere ancora un outsider. Semmai, ho cercato e cerco di destabilizzare dall'interno: anche se so che la macchina mi schiaccerà, cerco di mandarla fuori giri. (Lipperini 52)

Con questo romanzo, quindi, Scurati ammette di volere destabilizzare il sistema dall'interno per mandarlo "fuori giri" (Lipperini 52). Similmente, il professore alter ego de Il bambino che sognava la fine del mondo ammette di aver partecipato, numerose volte, al programma televisivo Matrix, soprattutto in puntate dedicate ad episodi di cronaca nera, per criticare "l'eccesso di attenzione riservato a quei fatti dai media" (216). Così come l'autore Antonio Scurati viene criticato da Scarpa, anche gli amici e i colleghi del professore-narratore lo rimproverano dicendogli che "cercare di usare la televisione contro se stessa [...] era una pia illusione nella migliore delle ipotesi, e una pelosa furbizia nella peggiore" (216). Anche in altre opere, come ad esempio ne Il sopravvissuto, Scurati mostra tutto il suo interesse per i media in relazione soprattutto ad eventi tragici tanto che sembra affascinato dal disastro. Ne Il sopravvissuto, però, il massacro compiuto da Vitaliano Caccia precede l'evento mediatico. Ne Il bambino che sognava la fine del mondo, al contrario, la tragedia inizia proprio quando la televisione punta i suoi riflettori sulla città di Bergamo, i cui abitanti soffrono di quell'inesperienza che Scurati ha ampiamente commentato nel suo saggio La letteratura dell'inesperienza.

Nel prologo a Il bambino che sognava la fine del mondo il protagonista narratore presenta brevemente gli eventi attorno ai quali si svolgerà la sua narrazione. Le pagine che seguono il prologo vogliono essere il resoconto dei fatti accaduti a Bergamo tra il 25 giugno 2007 e il 13 giugno 2008. Questi accadimenti narrano di 
una "pestilenza dell'anima e delle menti" (9). Nel romanzo, infatti, si parla di ripetuti episodi di pedofilia che coinvolgono don Mariano Presti e tre insegnanti della scuola materna "Gianni Rodari” nel centro di Bergamo: Niva Beghetto, Maria Pellegrini e la cittadina nigeriana Mati Wangari. Questa trama prende spunto da episodi di abusi sessuali che hanno coinvolto realmente la scuola materna "Olga Rovere" di Rignano Flaminio, un piccolo paese nei pressi di Roma, e don Pierino Gelmini (20 gennaio 1925-12 agosto 2014), fondatore dell' istituto per il recupero dei tossicodipendenti "Comunità Incontro." ${ }^{10}$ In un'intervista rilasciata a Borgato, Scurati sottolinea che l'intento del libro, però, "non è tanto quello di sondare l'entità degli episodi di pedofilia dell'istituto di Rignano Flaminio, quanto di trarne spunto per una riflessione sulla realtà dell'informazione oggi" ("Intervista"). ${ }^{11}$ Va qui evidenziato, inoltre, che con il romanzo Il bambino che sognava la fine del mondo, Scurati risulta essere in anticipo di più di tre anni rispetto alla sentenza definitiva del caso di Rignano Flaminio; ${ }^{12}$ infatti, dopo sei anni di indagini e di processi, il 28 maggio 2012 tutti e cinque gli imputati del caso Rignano sono stati assolti per insussistenza di prove. ${ }^{13}$ Già nel 2009 Antonio Scurati aveva assolto gli imputati coinvolti nel suo caso, il "caso Bergamo", incoraggiando il lettore a ulteriori riflessioni sul complesso rapporto che si viene ad instaurare tra finzione e realtà in un mondo dominato prevalentemente dalla cultura dei mass media.

Il bambino che sognava la fine del mondo non va inserito solo tra quei componimenti misti di finzione e cronaca, ma anche all'interno di opere di autofinzione ${ }^{14}$.

\footnotetext{
${ }^{10}$ Il processo a don Pierino Gelmini era stato sospeso a causa delle gravi condizioni di salute dell'imputato. Cfr. "Don Gelmini, processo sospeso", La goccia. Una finestra sull'Umbria, 29 maggio 2012, «http://www.lagoccia.eu/cronaca/8946-don-gelmini-processo-sospeso.html», consultato il 19 ottobre 2013. Nel frattempo, l'imputato è deceduto.

${ }^{11}$ Scurati commenta questi episodi di cronaca anche nei due saggi "Chi ha paura dell'uomo nero" (61-69) e "L'estate dei preti pedofili" (91-95) presenti nella raccolta Gli anni che non stiamo vivendo pubblicata nel 2010 da Bompiani.

${ }^{12}$ Il bambino che sognava la fine del mondo è apparso in libreria il 18 marzo del 2009. La sentenza definitiva del caso di Rignano Flaminio risale al maggio 2012, anche se gli indagati erano già stati scarcerati nel maggio del 2007. Contro questa scarcerazione era ricorsa in Cassazione la procura di Tivoli (Scurati, Gli anni 66).

${ }^{13}$ Cfr. "Abusi scuola Rignano: assolti tutti: non ci fu violenza sui bambini”, Il messaggero, 28 maggio 2012, «http://www.ilmessaggero.it/roma/cronaca/abusi_scuola_rignano_assolti_tutti_ non_ci_fu_violenza_sui_bambini/notizie/199019.shtml», consultato il 19 ottobre 2013.

${ }^{14}$ Giglioli definisce l'autofinzione come "stella polare della vasta galassia della non fiction" (53).
} 
Onde evitare fraintendimenti, in un'intervista, Scurati sottolinea la diversità tra un'opera autofinzionale ed una autobiografica; cosciente delle somiglianze con il suo personaggio, lo scrittore afferma che quella narrata "[è] la mia vita, ma è raccontata con la libertà che si concede l'autore di un romanzo di invenzione" ("Retorica”) e ribadisce che:

[i]l protagonista di questo romanzo non viene mai nominato, ma assomiglia moltissimo a me, perché fa le cose che faccio io. Lo si capisce dal fatto che, a un certo punto, accadono a questo alter ego delle cose che sono evidentemente romanzesche. ("Retorica”)

Nella stessa intervista, Scurati sostiene di voler ribadire questa differenza tra autofinzione e autobiografia per rassicurare i propri genitori e la sua compagna, "una delle persone più incattivite dall'apparizione di questo libro [...]". È evidente che lo scrittore si fonde facilmente con il suo alter ego finzionale, anche se, nel romanzo, il professore universitario resta senza un nome proprio. Come Scurati, però, anche il personaggio finzionale è docente universitario e opinionista per il quotidiano "La Stampa". Nel romanzo, inoltre, abbondano le riflessioni del professore sulla quotidianità e su quegli eventi di cronaca che hanno travolto la società, soprattutto italiana, negli ultimi vent'anni. Il professore riflette sull'insoddisfazione dei baby boomers, sullo sfacelo dell'università italiana, sulla violenza della cronaca, sui PACS, sul tasso di natalità in Italia, sulla pedofilia e, persino, sulla pedofobia. Le stesse considerazioni del romanzo sono presenti anche nella raccolta Gli anni che non stiamo vivendo. Il tempo della cronaca ed alcune di queste riflessioni sono anche apparse su quotidiani italiani. Proprio per questo, alla fine del volume Gli anni che non stiamo vivendo Scurati ringrazia i direttori dei giornali che "in questi anni hanno ospitato molti dei testi qui riprodotti, talora in forma diversa” (305) e, si dovrebbe aggiungere, a volte, riportati verbatim. È qui inutile rintracciare il percorso seguito da questi testi, ovvero quale sia apparso prima e dove; ciò che va comunque evidenziato è il formarsi, nella finzione narrativa, di un'immagine inscindibile tra Antonio Scurati e il suo protagonista, tanto da riuscire a creare sconcerto persino ai genitori ed alla compagna dello scrittore. E questa immagine coincide anche con quella formatasi attorno all'autore e creata dall'autore stesso grazie alle sua regolare partecipazione a programmi televisivi in cui si presenta anche per promuovere i propri romanzi. Basti qui ricordare 
Lettere dal Nord, una rubrica che si offre come uno sguardo sull'attualità italiana. Per questo motivo, il professore del romanzo, io narrante e protagonista, agisce indisturbato all'interno di una nebulosa dai confini incerti in cui il reale, il fittizio e il mediatico entrano in contatto sfiorandosi vicendevolmente. Questa commistione è rafforzata abilmente anche dalla presenza del bambino che dalla copertina del libro sembra eludere timidamente lo sguardo del lettore.

Non serve certo la conferma da parte dello scrittore per capire che il bimbo biondo dagli occhi azzurri ritratto sulla copertina è proprio Antonio Scurati. Non solo il professore del romanzo assomiglia molto a Scurati, ma anche le esperienze di quel bambino "che sognava la fine del mondo" presente nei corsivi del romanzo si possono ricollegare ampiamente ai primi anni di vita dell'autore stesso. Scurati dichiara che, in quest'opera, la sua infanzia gli è venuta in soccorso e che "i capitoli in corsivo che trattano di incubi, episodi di sonnambulismo e terrore nell'età in cui si è più deboli e esposti, fanno parte della mia storia personale" (Scurati, "Intervista"). Nel romanzo, la narrazione del professore si alterna ripetutamente a pagine scritte in corsivo in cui si legge di un bambino particolarmente suscettibile che soffre di una forte forma di sonnambulismo. Questo bambino, con l'alter ego di Antonio Scurati, non fa che accentuare il carattere autofinzionale del romanzo. Come ci ricorda Daniele Giglioli in Senza trauma, l'autofinzione è "un testo in cui per contratto non si può mai prescindere dall'imago, se non dalla figura reale, di chi scrive" (53); per questo le parole scritte dall'autore vengono percepite dal lettore come se fossero dette "da una voce reale, da una persona concreta che risponde con nome e cognome" (Giglioli 53) a quella dell'autore stesso. A conclusione del prologo (9-14), il professore sostiene di essere stato coinvolto in prima persona in questa vicenda di pedofilia. Nonostante una pretesa di autenticità, l'autofinzione mette anche in risalto l'evanescenza dei confini tra realtà e finzione (Giglioli 24). Analogamente, secondo Mazzarella, "[n]on esiste nessuna testimonianza che possa garantire alla realtà un sostegno inoppugnabile" (27); non a caso, la testimonianza del docente universitario comincerà presto $\mathrm{a}$ farsi sempre più labile. Più il professore si addentra nel caso di Bergamo, più le sue certezze vacilleranno; per questo l'uomo adulto si ripiega sempre più sul bambino in cerca di verità.

Antonio Scurati non ci offre semplicemente la rielaborazione romanzesca di due storie di pedofilia, ma ci presenta un'affascinante spiegazione di come una macchinazione possa essere montata, prima attraverso i giornali, e poi, su scala 
nazionale, grazie alla televisione e all'osceno" ${ }^{15}$ "spettacolo televisivo orrorifico" 16 a cui si assiste quotidianamente in nome dell'informazione. Similmente, anche il professore protagonista si ripropone non solo di narrare i "meri fatti" (14) accaduti a Bergamo, ma soprattutto, di raccontare "un'invocazione" (14) e "un silenzio" (14). Questa macchinazione mediatica trova un terreno fertile in cui attecchire e prosperare nella Bergamo benestante di inizio millennio. Va qui evidenziato che le accuse di pedofilia, mosse a don Mariano Presti e alle tre maestre, crolleranno a fine libro dopo l'ammissione di Marisa Comi, madre della supposta vittima, di essersi inventata tutto. Ospite a Matrix, popolare programma televisivo di approfondimento giornalistico, la donna, alquanto scossa, farfuglia di essere una "bugiarda e una malata" (272) e di avere "mentito su tutto" (272). La stessa signora Comi ammette di non sapersi spiegare come una tale montatura abbia potuto attecchire senza essere stata smascherata. ${ }^{17}$ Dopo questa confessione, ci si potrebbe legittimamente domandare se Comi stia nuovamente mentendo; del resto, lei aveva pur scoperto "delle gocce di sangue sulle mutandine di sua figlia" (272). Queste perplessità verso la dichiarazione spontanea di Comi colgono sia il lettore che alcuni cittadini di Bergamo i quali continuano a credere nella colpevolezza delle maestre. Antonio Scurati crea abilmente un clima di sospetto e di complotto, fomentato dalla cronaca e dai media, in cui la veridicità delle testimonianze viene messa continuamente in dubbio. Inoltre, lo scrittore ci mostra come la cronaca sia riuscita ad irrompere violentemente nella vita quotidiana tanto da trasformare il ventunesimo secolo nel secolo della "violenza della cronaca" (Il bambino 41). La cronaca, a differenza della storia, insiste sul presente, sul momento; i media, infatti, rendono "accessibili in tempo reale esperienze di vita violenta e di violenza estrema" (Letteratura 45) che coinvolgono individui in ambienti lontani dallo spettatore. La cronaca, quindi, non spiega l'evento all'interno di un quadro più ampio, ma lo abbandona a se stesso, isolato, e alla sua insignificanza. Per questo,

\footnotetext{
${ }^{15}$ Per Giglioli l'osceno "è ciò che non dovrebbe essere detto, ciò che dovrebbe sempre e comunque restare fuori scena" (24).

16 "Lettere dal Nord" in Parla con me, «http:///www.youtube.com/watch?v=MxrwWmCZ9Lg", consultato il 7 ottobre 2013.

${ }^{17}$ La rivelazione della Comi provoca stupore, sgomento e incredulità nel pubblico e trasforma il talk show in un "reality show, cioè verso la rissa, l'incidente, il colpo di scena [...]" (Travaglio 64). Secondo Travaglio questo è il formato verso cui tendono i talk show italiani. I nostri "talk-reality show" non sono condotti da "scintillanti David Lettermann e Jay Leno" (64), ma "dal mortifero Vespa, dalla balbettante La Rosa, [...] dall'inutilmente sorridente Mentana [...]” (64).
} 
i cittadini di Bergamo ricorrono alla superstizione ${ }^{18}$ nel tentativo di interpretare quegli accadimenti che hanno scosso la loro città; con il passaggio dal tempo della storia, pieno di contenuto, a quello della cronaca si perderebbe "soprattutto la possibilità di dare un senso alle nostre sofferenze. Di qui la superstizione del Male" (Scurati e Vattimo, "Conversazione”).

I casi di pedofilia discussi nel romanzo vengono prima presentati separatamente; inizialmente, don Mariano viene accusato da alcuni tossicodipendenti che sono passati dalla sua "Comunità Incontro". Appena la notizia viene fatta circolare, Massimo Gramellini, vicedirettore de "La Stampa", contatta il professore per chiederne un commento e lo esorta, calorosamente, dicendogli: "Tu sai scrivere. La notizia c'è, la storia anche, devi solo metterci sotto la tua penna" (55). Attraverso le abili parole di un intellettuale, suggerisce Gramellini, si può montare un caso di interesse nazionale. Proprio dalle accuse a Don Mariano prende spunto Marisa Comi, moglie insoddisfatta di un "gran puttaniere" (77) di provincia, che denuncia per pedofilia tre maestre della scuola "Rodari". Questa, alla fine del romanzo, è la confessione della donna attraverso le parole del narratore:

In città circolavano voci sui preti pedofili [...], qualcuno diceva ci fossero addirittura due maestre pedofile [...]. Perfetto. Le avrebbero creduto. [...] La gente pensa sempre al peggio. Aspettavano soltanto che qualcuno scagliasse la prima pietra. (273)

Ed una volta scagliata la prima pietra ha inizio una cascata di denunce che coinvolgono ben undici famiglie e quattordici bambini abusati. Qui la suggestione di massa fomenta l'isteria di massa causando forti disturbi tra i più vulnerabili, ovvero i bambini. Come sostiene Robert Bartholomew, la suggestione di massa non causa alcun sintomo di malattia; essa semplicemente alimenta la diffusione rapida, spontanea e temporanea di credenze false ma plausibili all'interno di una comunità (Bartholomew 12). L'isteria di massa, al contrario, provoca sintomi fisici nelle sue vittime: "[i]t is assumed that the victim is reacting to a state of extreme psychological conflict by unconsciously converting the conflict into physical symptoms" (Bartholomew 30). A Bergamo la suggestione di massa prima genera

\footnotetext{
${ }^{18}$ Andrea Di Consoli definisce giustamente Il bambino che sognava la fine del mondo come "romanzo superstizioso", e si domanda se questo non sia altro che "un rito arcano [...] tutto costruito per un bambino che sta per nascere" (87). Infatti, il romanzo è dedicato "[a] te che stai arrivando. Che tu sia la benvenuta a questo mondo" (5).
} 
isteria e poi la rafforza mescolandosi a essa. I genitori dei bambini sono vittime di suggestione e, quindi, in loro non è rintracciabile alcun sintomo fisico; le loro paure, però, travolgono i più piccoli, i bambini della scuola materna "Rodari", che manifestano veri e propri disagi e sintomi fisici, compatibili verosimilmente con i sintomi tipici di abusi sessuali. Il venti novembre 2007 si svolge un'assemblea tra i genitori degli alunni, la preside della scuola "Rodari" e la dottoressa Sommadossi. Dopo aver sentito l'elenco dei sintomi tipici che possono svelare abusi sessuali, Scurati commenta ironicamente che "sulla base di quei criteri diagnostici, qualsiasi bambino poteva essere sospettato di aver subito abusi sessuali. E anche parecchi adulti” (92). L'intera comunità bergamasca viene colta dal panico ed è preda di una vera e propria allucinazione di massa dove ogni singola persona è un potenziale pedofilo $^{19}$ e il sospetto si diffonde facilmente ovunque. Questo contagio è possibile proprio perché l'immaginario è diventato oggi, nella cultura di massa, una forza collettiva e non più individuale, e l'immaginazione si è trasformata in un "campo organizzato di pratiche sociali" (Scurati, La letteratura dell'inesperienza 67). Inoltre, come scrive Giglioli, “[p]ullula ovunque un'immaginazione del disastro spicciolo” (18). Per questo, la menzogna di Marisa Comi dà origine ad un'epidemia del terrore che miete delle vittime reali. I bambini, infatti, soffrono realmente: hanno incubi, urlano nella notte, diventano aggressivi e smettono di mangiare. La bambina Margherita Comi viene ritratta in un video mentre, addormentata, lancia un grido straziante, simile al "vagito della sirena di un bastimento" (94): "L'urlo agghiacciante non ha svegliato la bambina. Urlo e urlatrice sono una cosa sola. Indistinguibili" (94). Riflettendo su questo, il professore narratore sostiene che tale urlo può essere stato causato solo da sofferenze reali: in quell'urlo "ci doveva essere qualcosa di vero, oppure non c'era niente di vero al mondo. Non si poteva urlare così se non si era stati feriti” (99).

Nel romanzo viene illustrato un clima apocalittico che investe l'intera città di Bergamo, soprattutto dopo la fuga di notizie apparse su Internet secondo cui "una setta di satanisti stuprava i bambini al riparo delle mura del Seminario Vescovile, con la complicità delle maestre" (138). Nel giro di poche ore questa notizia passa di bocca in bocca come "un rantolo convulso" (138) fino a raggiungere un raggio ed un’intensità spropositati una volta approdata sui giornali e in televisione.

\footnotetext{
${ }^{19}$ Questo clima è simile all'atmosfera descritta da Truman Capote nel suo famoso romanzo In Cold Blood. Una volta scoperti i cadaveri della famiglia Clutter, gli abitanti di Holcomb cominciarono ad osservarsi con sospetto e "[...] old neighbors viewed each other strangely, as strangers" (5).
} 
Nel ventunesimo secolo, ci si trova di fronte ad una vera e propria caccia alle streghe echeggiante i processi di Salem, Massachusetts, di fine diciassettesimo secolo e simile alla persecuzione narrata da Giorgio Vassalli ne La chimera. Nell'odierna e prospera Bergamo la paura causa effetti deleteri che fanno regredire parte della popolazione ad antiche superstizioni. Bartholomew sostiene che "[m] odern-day child-molestation or satanic-cult fears resemble the persecutions of various deviants and ethnic groups during medieval European witch-hunts" (24). A distanza di secoli, quindi, continuano a persistere paure primordiali; per questo, a Bergamo, persino la pedofilia viene assimilata a una setta e a macabri riti satanici. In città, infatti, non si parla di pedofilia come di una perversione del desiderio sessuale, bensì come di una manifestazione del Male e, di conseguenza, si va invocando l'apocalisse. In realtà, all'interno di un gruppo, si rintracciano erroneamente abusi sessuali sui bambini, in connessione con riti satanici, quando il tessuto morale della comunità si fa sempre più labile e le famiglie stesse si sentono minacciate e incapaci di proteggere i propri figli. Tali suggestioni si diffondono più facilmente in periodi di inquietudine sociale e di recessioni economiche (Bartholomew 20).

Le vicende de Il bambino che sognava la fine del mondo si svolgono tra il 2007 e il 2008, ovvero proprio agli albori di una grave crisi economica che ha colpito l'Italia e l'intero sistema capitalistico occidentale a cui essa appartiene. Nella provincia bergamasca, infatti, gli imprenditori cominciano lentamente a delocalizzare le proprie fabbriche in Romania "per potersi approvvigionare di carne fresca a buon mercato" (156) e nella speranza di tamponare gli effetti disastrosi della recessione. Apparteniamo, secondo Scurati, all'umanità più longeva, sicura e agiata di tutti i tempi; nonostante questo, nel mezzo di una crisi economica destabilizzante, ci lasciamo prendere da una "pauretta collosa" che ci fa "vivere con il fiato corto" ("Retorica"). Questa "pauretta collosa” è la stessa paura su cui Bauman riflette in Paura liquida. Essa è ubiqua, ma difficile da identificare: "La paura più temibile è la paura diffusa, sparsa, indistinta, libera, disancorata, fluttuante, priva di un indirizzo o di una causa chiari [...]" (4). Questa paura è la paura che investe la nostra società liquida e si autoalimenta da sola.

I genitori dei bambini molestati formano un comitato e indicono una riunione presso la scuola "Rodari"; il volantino che esorta alla partecipazione degli adulti a tale incontro recita: "GLI ORCHI SONO TRA NOI. PROTEGGIAMO I NOSTRI BAMBINI” $(76)^{20}$. Uomini e donne adulti incominciano così la loro

\footnotetext{
${ }^{20} \mathrm{Nel}$ romanzo anche Mentana usa lo stesso linguaggio adottato dai genitori e, in una puntata di Matrix, rivolgendosi al professore chiede: "[...] Dobbiamo attrezzarci per andare a caccia di
} 
caccia agli orchi, che, come sappiamo, sono creature appartenenti all'immaginario infantile. Questi genitori attingono dalle fiabe il vocabolario necessario per parlare di pedofilia anche quando si rivolgono ad un pubblico adulto. Durante questo incontro viene mostrato un video di Margherita Comi, la prima presunta vittima. Questo video riprende la bambina che, sollecitata dalla madre e dalla zia, mostra quello che le maestre e l' "uomo nero" (96) le avrebbero fatto. Margherita prende l'asciugamano e "[c]omincia a sfregarselo su e giù. [...] Mima. Mima l'atto sessuale. Forse" (97). Altre due mamme girano un video analogo: qui si vedono un bambino e una bambina completamente nudi adagiati su un lettino. Spronato dalla madre il bimbo si getta sulla bambina e:

[1]e sfrega il bacino contro l'osso pubico. Lui simula l'atto sessuale come può farlo un bambino di cinque anni (il movimento desultorio di un verme di una ventina di chili), lei piange davvero. Una scena pornografica di amore umano, ma immaginata da un cane. (187) [corsivo nostro]

Non esistendo alcuna registrazione dell'abuso perpetrato dalle maestre e da Don Mariano, ci si accontenta di video girati tra le sicure mura domestiche, in cui l'atto sessuale viene simulato sotto la sollecitazione degli adulti.

Come ci ricorda Pavel, una simulazione può trasformarsi "in un atto effettivo pari a quello vero" (37); in questo caso, gli atti simulati non sono più distinguibili da quelli effettivi. Chi simula una malattia, ad esempio, può veramente finire a letto e determinare in sé qualche sintomo della malattia stessa (Baudrillard, Simulacri 61).

Questi genitori spaventati incentivano la simulazione creando, di conseguenza, dei veri e propri simulacri. I genitori girano i video per mostrarli poi nelle trasmissioni televisive in cui appaiono nella parte di "testimoni oculari". Secondo Baudrillard, viviamo proprio nell'era della simulazione e assistiamo alla continua "sostituzione al reale dei segni del reale" (Simulacri 61); le immagini, sempre più ad alta definizione, si sostituiscono al reale. L'immagine appartenente all'ordine della simulazione "è priva di rapporto con qualsivoglia realtà: è il puro simulacro di sé" (Baudrillard, Simulacri 66). Il simulacro è vero e non nasconde la realtà. Il

orchi, oppure stiamo solo conducendo una caccia alle streghe?" (223) Gli orchi appartengono al mondo fiabesco e le streghe a quello delle superstizioni; entrambi, quindi, non dovrebbero turbare il mondo reale degli esseri umani, o almeno quello degli adulti. 
filosofo francese si spinge perfino ad affermare che la simulazione, ad esempio, di un attentato aereo è tanto più grave dell'attentato stesso, in quanto la prima insidia il principio di realtà e sottolinea la mancanza di un referente, ovvero l'inesistenza dell'“equivalenza con il reale" (Simulacri 85). Per questo motivo, il professore, vedendo il video con il bambino di cinque anni simulante l'atto sessuale, parla di una vera e propria "scena pornografica di amore umano" (187) in cui la bambina "abusata" piange veramente. Proprio a causa della forza performativa della simulazione, i bambini della scuola "Rodari" soffrono realmente favorendo tra loro la diffusione dell'isteria di massa.

Senza offrire dettagli osceni, Antonio Scurati riesce efficacemente a far riflettere anche su un male silenzioso quale la pedofilia. Se quelli evidenziati nel romanzo sono solo alcuni dei sintomi riportati dai bambini che non hanno subito alcun abuso sessuale, com'è possibile concepire il trauma di quei bambini vittime vere di pedofili reali? ${ }^{21}$

Nei suoi scritti Scurati commenta spesso il proliferare di video e di immagini all'interno della nostra società che, grazie alla cultura di massa, assiste inebetita al "trionfo della visualità" (La letteratura dell'inesperienza 49). La televisione mostra regolarmente immagini di guerre, orrori e violenze; dal salotto di casa il telespettatore assorbe tali immagini che, pur essendo mediate e fortemente manipolate, generano "l'illusione dell'immediatezza" (La letteratura dell'inesperienza 54); simulando immediatezza, queste immagini mentono proprio sulla loro capacità di alterare la verità.

I video del caso di Bergamo non sono, però, gli unici ad assillare il protagonista del romanzo. In quei giorni, infatti, vengono trasmesse ossessivamente le immagini riprese da una telecamera a circuito chiuso nel parcheggio del centro commerciale Orio Center, prototipo del "nonluogo" di Marc Augé (7), in cui lo stesso professore, di tanto in tanto, si nasconde inebriandosi momentaneamente con acquisti inutili. Nel parcheggio di Orio Center si vede una zingara, Eleana Butinaru, sfondare il cranio a Maria Tirabassi, giovane madre di Clusone. La divulgazione senza tregua di questa scena non ha nulla a che fare con l'informazione,

\footnotetext{
${ }^{21}$ In Italia si stimano 21.000 casi di pedofilia all'anno. Cfr. «http://www.avvenire.it/shared/ pedofilia/dossierorchi.pdf", consultato il 10 novembre 2013. Antonio Scurati riporta dati ancora più allarmanti di uno studio del 2007 secondo il quale in Italia, ogni anno, i nuovi casi di violenza sui minori ammontano a 41.000. "Stando a questi dati, almeno un ragazzo italiano su sei sarebbe stato vittima di abusi sessuali nell'infanzia o nell'adolescenza” (Il bambino 91).
} 
visto che la zingara è già stata identificata, catturata e messa in galera. ${ }^{22}$ Più che di informazione, il narratore sostiene si tratti di "un rito collettivo di esorcismo del Male” (232), una vera e propria gogna mediatica. Questo video viene trasmesso in continuazione perché è in grado di catturare l'attenzione del telespettatore e ne soddisfa l'interesse morboso. Qui si potrebbero prendere in prestito le parole di Slavoj Žižek scritte per commentare le immagini diffuse ininterrottamente dalla televisione americana dopo il crollo delle torri del World Trade Center: "[...] we wanted to see it again and again; the same shots were repeated ad nauseam, and the uncanny satisfaction we got from it was jouissance at its purest" (12). Le immagini della telecamera a circuito chiuso di Orio Center costituiscono quello che Baudrillard definisce uno "pseudo-evento" (The Jean Baudrillard Reader 29): pur apparendo immediato e naturale, lo pseudo-evento è invece filtrato, manipolato e trasformato dai media in un prodotto mediatico finito e, quindi, esso può essere consumato come qualsiasi altro bene di scambio. Per questo motivo, è opportuno affermare che il giornalista, ai giorni nostri, non raccoglie più informazioni, bensì le crea producendo una merce ${ }^{23}$ all'interno di un collaudato sistema capitalista. ${ }^{24}$ Già negli anni sessanta "[n] ews gathering turned into news making” (Boorstin 14) e, di conseguenza, una notizia ben confezionata può, sempre più spesso, oscurare la pallida realtà. Per questo, il direttore Gramellini esorta il professore prima a scrivere un commento sulla vicenda di don Mariano (55) e poi sul caso delle mae-

\footnotetext{
${ }^{22}$ La messa in onda continua di questo video non fa che alimentare il mito dello "zingaro rapitore di bambini" (234) riportato, tra l'altro, alla ribalta nell'autunno 2013 dopo il ritrovamento in un campo nomadi greco di Maria, una bambina dai lineamenti nordici che, dopo il test del DNA, non è risultata essere la figlia della coppia di coloro che, inizialmente, avevano dichiarato di esserne i genitori. Cfr. "Grecia, caso della bambina bionda trovata tra i rom", «http://www. corriere.it/esteri/13_ottobre_22/grecia-caso-bimba-bionda-trovata-rom-traferiti-impiegatianagrafe-c9b40274-3af5-11e3-95f2-9a7a296f615f.shtml», consultato il 22 ottobre 2013. Il mistero della bambina è stato risolto nel giro di pochissimi giorni. Il caso è stato smontato velocemente. Maria, infatti, sarebbe stata "donata” ai rom greci da un'altra famiglia rom bulgara. ${ }^{23}$ Si rimanda qui allo studio di Daniel Boorstin A Guide to Pseudo-Events in America che fornisce un'interpretazione accattivante del sistema d'informazione americano.

${ }^{24}$ All'inizio del romanzo l'autore riflette sul capitalismo e sulle trasformazioni subite dalla pianura Padana, "terra che, nel volgere di pochi anni, aveva cambiato colore" (20) e le cui coltivazioni si erano adeguate alla domanda del mercato. In questa pianura, dove tutto va veloce, non si possono più piantare alberi lenti nella crescita e, quindi, lenti anche nella produzione di capitale. Per questo nella pianura Padana si dice addio agli alberi come il noce e la quercia e si accolgono con entusiasmo le arachidi che bevono poco e rendono molto (Il bambino 20-21).
} 
stre della scuola "Rodari" (86). Visto che "[...] il "caso Presti" non era "montato"” (86), Gramellini e Vidotto, abile cronista di nera, spronano il docente universitario ad occuparsi della scuola "Rodari" augurandosi che si possa così confezionare uno pseudo-evento vendibile alla folla. Poco importa quale sia la vicenda su cui scrivere; ciò che conta è che il professore scriva in modo convincente e che il caso da lui narrato monti, facendo sensazione e catturando l'attenzione del pubblico.

Le notizie si moltiplicano sui giornali e alla televisione; gli pseudo-eventi si mescolano agli eventi reali creando un vortice eccessivo d'informazione che produce disinformazione e, soprattutto, terrore. Gli pseudo-eventi sono prodotti dal desiderio dell'homo videns di mettersi in mostra, dall'urgenza di trasformarsi da telespettatore in attore:

Il dover «far vedere» ingenera poi il desiderio di «farsi vedere». Il che produce lo pseudo-evento, l'evento che avviene solo perché c’è una cinepresa che lo registra, e che non avverrebbe altrimenti. Lo pseudo-evento è dunque l'evento fabbricato per la televisione e dalla televisione. (Sartori 57)

Come sostiene Marshall McLuhan, la nostra epoca assiste al crollo delle barriere: "Our time is a time for crossing barriers, for erasing old categories-for probing around" (10). Nel romanzo Il bambino che sognava la fine del mondo i poli contrari si dissolvono: i genitori dei bambini cominciano ad apparire in televisione con $\mathrm{i}$ loro video amatoriali o intervistati dai giornalisti; loro parlano del proprio dolore per poi mettersi da parte ed assistere alle sofferenze di altri genitori. L'assemblea organizzata presso la scuola "Rodari" sfocia nella più totale confusione e il teatro dell'isteria di massa non riesce a sopportare "la divisione tra scena e platea" (93) e l'assemblea si trasforma in teatro puro dove la quarta parete viene abolita.

Il 12 dicembre del 2007 i genitori che accompagnano i loro figli a scuola credono di essere preda di un'allucinazione vedendo i carabinieri del RIS, "gli stessi dell'omonima serie tv" (106) recintare l'edificio dell'istituto con il nastro fosforescente. Questi genitori "dovettero credere di aver acceso la televisione. Ancora non sapevano che era la televisione che stava per accendersi su di loro" (107). L'annuale recita natalizia si svolge a porte chiuse nel giardino della scuola; qui i genitori si accalcano alle inferriate per assistere alla performance dei figli: "[s] tavano fuori, ma gli animali in cattività erano loro" (122) e non i loro bambini. 
In questo romanzo le strutture duali polari svaniscono lasciando spazio all'indeterminazione, alla confusione tra attore e spettatore e tra attivo e passivo. Baudrillard sostiene che ai giorni nostri, con la presenza massiccia dei mass media, "[n] on siamo più spettatori, ma attori della performance, e sempre più integrati nel suo svolgimento" (Il delitto perfetto 33). Il professore si ritrova persino nel ruolo di comparsa di fronte a Margherita Comi, che, protetta da un vetro a specchio, osserva sei uomini per identificarne un unico colpevole, ovvero colui che avrebbe abusato di lei. Proprio in questa occasione, l'alter ego di Antonio Scurati ha l'impressione di essere all'interno di un film di cui però non riesce a rammentare il titolo. Con un cartellino raffigurante un cavallo, lui partecipa ad un riconoscimento all'americana e comincia a tranquillizzarsi solo nel sapersi "in una situazione da film" (249). "Anche quella, in fondo, era una scena già vista. Non avevo nulla da temere. Ero già stato anche lì (249), così osserva il narratore-attore mentre se ne sta immobile in una stanza soffocante illuminata da fari accecanti. Il docente universitario non sembra turbarsi persino quando viene identificato da Margherita come l'uomo che le aveva fatto del male. Il culmine dell'unione e della confusione tra attore e spettatore viene raggiunto a fine romanzo, quando la signora Comi si confessa e si autoaccusa; lei getta uno sguardo di complicità e estraneità sul pubblico di Matrix e il suo sguardo si rivela essere "su entrambi i versanti della telecamera. Uno strabismo losco, un occhio alla scena e uno alla platea" (275). Tutto ciò rientra perfettamente nella sopraddetta abolizione dei poli contrari, dove palcoscenico e platea, attori e spettatori, vittime e carnefici, accusatori e accusati, eventi e pseudo-eventi, ed anche finzione e realtà abbattono le barriere creando una nuova dimensione.

In questa incontrollata espansione di immagini, digitali e non, il professore prova sollievo assistendo personalmente ad una fellatio (159): davanti ai suoi occhi e a quelli di una telecamera posta per scoraggiare la prostituzione, il protagonista vede, nell'abitacolo di una macchina, una giovane ragazza che procura piacere ad un uomo maturo. Questa, commenta il narratore, è "la vita vera, nuda e cruda" (159). Tra mille simulazioni e docufiction questa scena appare in tutta la sua convenzionale realtà $e$, per questo, gli provoca una consolazione temporanea. Tale conforto, comunque, non è destinato a perdurare nel tempo: cessa, infatti, quando la mente dell'intellettuale comincia a meditare sulla prostituzione. Questa ragazzina, seppure in carne ed ossa, si smaterializza "nel simulacro di una fantasticheria erotica" (161), dove il rapporto sessuale equivale a "derealizzare, smaterializzare, annichilire" (161). Ed è proprio questa "scomparsa degli oggetti nella loro stessa rappresentazione” (Baudrillard, Simulacri 24) che trasforma la realtà in iperrealtà. 
Queste telecamere, installate nel quartiere degli immigrati, non avrebbero fatto altro che produrre "un sovrappiù d'eccitata osservazione" (160) in un paese di "ipocriti guardoni” (161). Come sostiene Baudrillard, ai giorni d'oggi "[n]ulla sfugge, c'è sempre una videocamera da qualche parte” (La scomparsa 12) pronta a riprenderci; infatti " $[\mathrm{s}] \mathrm{i}$ può essere filmati a nostra insaputa ed essere chiamati a recitare nuovamente tutto ciò in una rete tv" (La scomparsa 12). Il filosofo francese si spinge a sostenere che "la videocamera virtuale è nella nostra testa" (La scomparsa 12). All'aeroporto di Milano Linate, infatti, il professore sembra possedere degli occhi meccanici:

Ogni singola persona, ogni singolo oggetto presente nell'immensa sala dell'aerostazione di Linate mi feriva la retina. Era calato su di me il dono profetico della visione periscopica [...]. Percepivo con traslucida evidenza ogni minimo dettaglio della realtà circostante [...]. (264)

Anche all'interno dello spogliatoio del Seminario, in cui si è introdotto sentendosi come "un agente segreto sotto copertura" (Il bambino 61), il docente universitario sembra osservare la realtà attraverso la lente di una videocamera. Prima, in piscina, nota un seminarista di colore dotato della "proverbiale possanza fisica dei neri nubiani" (61); del seminarista riprende i "poderosi colpi di reni" (61) in un "aggraziato e violento movimento sussultorio" (61). Nello spogliatoio, lui si ritrova, seduto, proprio all'altezza del membro del ragazzo di colore che, in un istante, si è levato il costume bagnato; del membro maschile il narratore fornisce una descrizione ad "alta definizione". Quest'organo, enorme e sproporzionato rispetto al resto del corpo, "[s]ebbene a riposo, appariva enfio e tonico. La sua consistenza era tale da dare una singolare impressione di autonomia rispetto al resto del corpo, come se vivesse di vita propria" (63). Esso appare come "carne debordante" e "carne sessuale" (61). A questo punto non ci si dovrebbe stupire di poter penetrare all'interno del corpo del seminarista attraverso una videoendoscopia. Il professore conclude il suo primo ed ultimo sopralluogo nel seminario riflettendo su quanto osservato e sovrapponendo l'immagine con il suo referente, la carne. Riflettendo sul membro del seminarista, il narratore osserva:

Mi parve la cosa più singolare che avessi visto da molto tempo a quella parte: era carne sessuale, vale a dire quanto di più materiale si potesse 
concepire, e al tempo stesso un puro simbolo [...]. Era un'immagine rappresentativa e al contempo la cosa rappresentata: un tatuaggio di carne su carne. (63) [corsivo nostro]

Anche qui, come nel caso della baby-squillo sopraccitata, la dialettica tra immagine e realtà viene annullata e il materiale si smaterializza nel suo simbolo.

Accanto alle immagini di giornali, telegiornali e reportage e come complemento di queste, la televisione italiana si è lasciata prendere, negli ultimi vent'anni, da un'"ideologia di un nuovo realismo" (83), che produce anche delle docufiction, come, ad esempio, quella intitolata Erba. I giorni dell'odio andata in onda, in prima serata, su Canale 5 nel 200725. Proprio in queste pagine dedicate al caso di Erba, il narratore lancia una pungente critica, ai limiti della parodia, verso la televisione italiana ed i suoi sempre più numerosi programmi reality che, ben lontani dal creare informazione, alimentano oscenità, sensazionalismo e terrore, facendo leva su un pubblico fortemente suscettibile. Mancando Rosa Bazzi, casalinga assassina di Erba, ci si può sentire appagati semplicemente da Paola Messina, l'attrice interpretante il ruolo dell'omicida. ${ }^{26}$ Questo, secondo l'autore, è un realismo di “fuochi fatui” (83), una iperfinzione in cui prevale ciò che ci illude di essere reale:

Da una quindicina d'anni a quella parte, l'ideologia di un nuovo realismo si era impossessata della televisione, e di lì era dilagata ovunque. Si trattava, però, di un realismo di fuochi fatui su tombe scoperchiate: in esso trionfava non ciò che si fingeva reale ma ciò che c'illudeva d'esserlo, e per farlo sostituiva l'osceno al tragico. Una iperfinizione che per darci prova della sua autenticità sprofondava

\footnotetext{
${ }^{25}$ Gli ascolti della docufiction voluta da Enrico Mentana all'interno di Matrix si sono aggirati attorno ai tre milioni e mezzo di telespettatori ( $16.5 \%$ di share). Il dibattito successivo, che ha coinvolto persino Azouz Marzouk, padre del bambino ucciso, ha registrato ascolti record pari al $27.46 \%$ di share. Apparentemente, tutto ciò è stato ordito con lo scopo di offrire "un buon prodotto giornalistico". Cfr. "Strage di Erba a Matrix. Fa più ascolti il dibattito della docufiction” «http://archiviostorico.corriere.it/2007/giugno/20/Strage_Erba_Matrix_piu_ ascolti_co_9_070620114.shtml», consultato il 24 ottobre 2013.

${ }^{26}$ Del caso di Erba si può sostenere quanto detto da Marco Travaglio sul caso di Cogne; ennesimo "diversivo" (34) offerto dalla televisione italiana per sviare l'attenzione pubblica dai processi ai politici di casa nostra.
} 
sempre più nei toni crudi della vita, nel sangue, nello sperma, rimestava nel torbido, nell'abietto. (83-84) [corsivo nostro]

Secondo l'autore il racconto offertoci dalla televisione ha una grande forza performativa e "fa accadere ciò che rappresenta, lo pone in essere dicendolo" ( $\mathrm{La}$ letteratura dell'inesperienza 56). Questo è possibile a causa della trasformazione del nostro mondo in "un mondo che comincia e finisce con la televisione" ( $\mathrm{La}$ letteratura dell'inesperienza 55) e alla mutazione dell'essere umano da "homo sapiens" a "homo videns." 27 Scurati ci mostra una società in cui l'essere umano si è trasformato da cittadino a voyer insaziabile, principalmente, del dolore altrui. Anche i genitori del caso di Bergamo registrano video amatoriali, le loro personali docufiction, nella convinzione di essere testimoni oculari dei presunti abusi subiti dai figli. Così facendo, questi genitori non fanno altro che alimentare l'isteria di massa, ponendo in essere quanto rappresentato. Le immagini, infatti, possono anticipare e precedere la realtà (Baudrillard, Evil Demon of Images 19). La rete mediatica, la televisione e la velocità con cui vengono diffuse le immagini recidono il legame millenario tra vedere e sapere; l'equazione "io vedo e quindi so" si è sgretolata in pochissimi anni. L'illusione però del contratto tra sensi e sapere rimane e per questo si vuole ancora vedere a tutti i costi. Non a caso, il professore, turbato da tutti i video simulati, si augura che venga trovato quello di una telecamera a circuito chiuso, in cui si vedono le maestre mentre conducono i bambini all'esterno per portarli a Bergamo Alta dove sarebbero poi stati sottoposti a riti e ad abusi sessuali. Il tanto paventato ma desiderato video non viene mai trovato, non si materializza. In tal modo, "[v]iene meno il legame ontologico tra immagine e realtà: ciò che esiste per i miei occhi è esentato dal possedere la proprietà dell'esistenza. L'essere diviene un requisito superfluo" (La letteratura dell'esperienza 52).

In un periodo storico in cui l'accesso alle notizie è immediato si è creato paradossalmente un clima di incertezza a causa dell'eccesso di informazione; nell'epoca delle immagini, le immagini si fanno sempre meno probanti. "Attraverso l'abolizione della distanza, del 'pathos' della distanza, tutto diventa indecidibile" (Baudrillard, Violenza del virtuale 12). Ciò crea scetticismo, credulità universale e "agnosticismo umorale", ovvero, "non si crede più a niente e ci va bene tutto" (Scurati, Letteratura dell'inesperienza 55).

\footnotetext{
${ }^{27}$ A tale riguardo si rimanda a Giovanni Sartori e al suo Homo videns.
} 
Quest'atmosfera di terrore e psicosi collettivi crea una tale confusione da portare l'intera comunità bergamasca ad immedesimarsi nel ruolo della vittima. Come ci ricorda Giglioli, l'essere umano contemporaneo anela ad essere qualcosa in quanto vittima di qualcun altro:

[...] l'identità contemporanea riesce a pensarsi solo tramite il dispositivo dell'identificazione vittimaria. Io sono ciò che ho subito. E se non ho subito nulla sono nulla. [...] Dove nulla e nessuno può più farci grande torto, guai a non avere torti da recriminare, e identità di vittime da rivendicare. (10)

Similmente, anche Baudrillard sostiene che "tutto il movimento di una società indifferente, termina nella vittimalità [...]" (Il delitto perfetto 135). In primo luogo, la comunità bergamasca si sente in pericolo e vittima di un indeterminato Altro, l'immigrato, di cui spesso non conosce neppure le origini. Gli immigrati, secondo alcuni abitanti del luogo, metterebbero in pericolo l'economia locale e praticherebbero oscuri riti di magia. La proprietaria di una merceria in via Zambonate spiega di dover terminare la propria attività per colpa degli immigrati cinesi. Parlando con il professore la commerciante afferma: "Chiudiamo perché con quelli là va tutto in malora. Vendono pezze e ci mettono gli schiavi a fabbricarle. Sono capaci di tutto quelli lì" (114). Similmente, il guardiano della scuola "Rodari" accusa gli immigrati di aver ucciso un gattino ritrovato nel giardino dell'istituto: "Sono capaci di tutto quelli lì. Anche di fare il vodoo" (43). ${ }^{28}$ Più di tutti gli altri, il ruolo di vittima viene consapevolmente scelto e abilmente interpretato da Marisa Comi, che, nella sua veste vittimale, diventa una vera e propria "celebrità" (148). Ospite a Matrix, però, Marisa Comi non riesce più a reggere tale ruolo e, per questo, svela il suo inganno ammettendo di avere inventato tutto. Trovato del sangue sulle mutandine della figlia, la signora Comi è pressoché "[...] impazzita. 'Bisogna dare la colpa a qualcuno!' aveva immediatamente intimato alla figlia. Lo aveva detto d'impulso, senza pensarci, sotto la spinta di un'urgenza irrefrenabile" (273). Una volta abbandonata dal marito, Comi non riesce più, da sola, a curare la

\footnotetext{
${ }^{28}$ Antonio Scurati sfrutta abilmente e a proprio vantaggio alcuni topoi ricorrenti della narrativa italiana odierna. A partire dagli anni Novanta, l'Italia si è trasformata in terra di immigranti e la figura dello straniero, anche se spesso stereotipata, ha preso sempre più piede nel romanzo italiano, nelle opere sia di scrittori italiani che di scrittori migranti. (Cfr. Elisabetta Mondello "Finzione narrativa ed 'effetto realtà", p. 15).
} 
crescita e l'educazione della figlia. Esaurita, a volte, sogna addirittura di soffocarla nel sonno, meditando, probabilmente, di emulare Annamaria Franzoni, un'altra protagonista di un fatto di cronaca nera, il delitto di Cogne, che ha diviso l'Italia intera tra colpevolisti e innocentisti. Per questo, la madre si convince che "se Margherita fosse stata vittima di qualcosa di terribile l'avrebbe amata di più, sarebbe stato più facile volerle bene, starle accanto" (273). Inoltre, "[e]ssere la mamma di quella bambina disgraziata l'aveva fatta sentire finalmente viva" (273).

La signora Comi vuole farsi sentire e, per questo, è alla ricerca di un suo trauma da raccontare, dato che "[t] rauma è oggi tutto ciò di cui si parla" (Giglioli 8). Marisa Comi, inoltre, desidera sentirsi viva proprio come Mati Wangari, che, a differenza di lei, è viva, "gigantesca e imperiosa" (245). Arrivata clandestinamente in Italia sulla rotta delle schiave, Mati Wangari era riuscita prima a diplomarsi e poi a laurearsi. Successivamente aveva trovato lavoro presso la scuola "Rodari", aveva fondato l'associazione Nuovo Mondo, aveva avviato un'attività commerciale di successo e, sposatasi con un connazionale, aveva pure trovato il tempo di mettere al mondo cinque bambini. Di fronte a tanta esuberanza, Comi riconosce la propria inadeguatezza e apatia e ammette di essere stata invidiosa della nigeriana Wangari (274). Nello stesso modo il professore comincia ad osservare sé stesso, ritornando al bambino che è stato. Lui rintraccia così i sintomi di una ferita non ben definita infertagli durante l'infanzia. Seguendo questo caso di pedofilia, il professore-scrittore si trova a pensare che "un qualche trauma dovevo averlo subito anch'io" (190). Il docente universitario si convince di essere stato vittima di un trauma ormai rimosso e per questo comincia a scavare nel proprio passato, andando persino a trovare i genitori nel tentativo di scoprire l'origine dei suoi forti turbamenti. All'inizio del romanzo le pagine in corsivo dedicate al bambino sembrano essere completamente separate dal resto del testo, dalla narrazione del professore; man mano che lui si lascia coinvolgere nel caso di pedofilia, invece, le esperienze del bambino cominciano ad emergere nell'uomo adulto turbandolo, creandogli dei forti scompensi psichici e gettandolo in preda alla paranoia. Così come le barriere tra realtà e finzione, spettatore e attore, vittima e carnefice si fanno sempre più labili, crolla anche la netta distinzione tra il bambino e l'uomo adulto, tra infanzia e età adulta. Proprio come la signora Comi, però, anche il professore, alla fine, deve riconoscere di non aver subito alcun abuso e di non essere mai stato molestato da bambino. Anche lui, appartenente ad una generazione agiata ma sofferente, è preda di uno stato vittimale. Successivamente deve comunque rendersi conto della totale infondatezza dei propri sospetti. A fine romanzo, 
infatti, sua madre lo rassicura che, da piccolo, nessuno gli aveva mai fatto del male, dicendogli: "Figlio mio, tu hai avuto un'infanzia normale. Urlavi nel sonno, giravi per la città di notte, ma non c'è mai stato niente di brutto nella tua infanzia. Credimi. Sei sempre stato suggestionabile. [...]" (266). Lei, però, ammette di aver pensato all'aborto durante la gravidanza e subito dopo questa sua rivelazione "sbadigliò" (267) lasciando il figlio "di sasso" (267). A questo punto il protagonista capisce che la malinconia, sua e della madre, può annullare il passato e alla fine, dopo aver represso il desiderio di abbracciarla e di cullarla, la saluta e ammette a sé stesso che "non c'era nessun altro dramma se non quello" (268), il dramma di una vita monotona in una società opulenta. In questo romanzo, l'autore ci offre un quadro desolante di una generazione "di privilegiati" che vivono "a stento" (Gli anni che non stiamo vivendo 5) in una società che, nonostante la crisi economica, è comunque caratterizzata da un benessere diffuso.

Antonio Scurati ha creato un racconto entusiasmante e un oggetto narrativo non-identificato in cui si mescolano "indifferentemente narrativa, saggistica e altro: prosa poetica che è giornalismo che è memoriale che è romanzo" (Wu Ming 12). Nel romanzo, infatti, la prosa giornalistica e la saggistica si saldano perfettamente ad altri passaggi più intimistici, come, ad esempio, le sentite riflessioni dell'autore sulla paternità. ${ }^{29}$ Ben lontano dal desiderio di offrire una semplice rappresentazione mimetica della realtà, Il bambino che sognava la fine del mondo fornisce un'avvincente, ma non irrevocabile, interpretazione della realtà e dei suoi meccanismi di costruzione. Questa illustrazione della società coeva è resa tanto più pungente e convincente dal fatto di essere stata elaborata proprio da uno studioso quale Antonio Scurati, un insider, che conosce a pieno il mondo mediatico italiano. Abilmente lo scrittore apre uno squarcio sulla realtà e, scrive Vattimo, induce il lettore a "un riconoscimento di ciò che già sa" (Scurati e Vattimo, "Conversazione"), ma che, prima della lettura, riusciva a stento a mettere a fuoco. Per questo Il bambino che sognava la fine del mondo è un romanzo che turba il lettore e lo mette a disagio.

Scurati interpreta alcuni casi di cronaca italiana e, grazie alla sua creatività, elabora un discorso illuminante sulla realtà, o meglio, sulla iperrealtà che, similmente ad un trompe l'ail, rende difficile tracciare una netta distinzione tra segno e referente. L'autore, inoltre, incoraggia una riflessione sullo stretto rapporto tra cronaca e letteratura. La cronaca è nel presente ed è fruita immediatamente dallo spettatore; la letturatura, invece, ha tempi diversi, più lenti. Per questo, afferma

${ }^{29}$ Queste riflessioni sulla paternità vengono rielaborate e ampliate nell'ultimo romanzo dell'autore, Il padre infedele (2013). 
Scurati, "una delle grandi sfide che la letteratura può raccogliere oggi è di misurarsi con il tempo della cronaca, scendere sul suo terreno" (Scurati e Vattimo, "Conversazione").

Il bambino che sognava la fine del mondo si confronta con la cronaca e, a volte, le assomiglia terribilmente, per poi, però, distanziarsi da questa e riportare il lettore nel tempo ampio della letteratura. La cronaca strepita fragorosamente; le notizie da cui siamo sommersi ci vengono date, confermate e smentite in continuazione. Alla fine del romanzo, il caso di Bergamo crolla e i giornali smontano l'evento: dopo la dichiarazione della signora Comi, tutto sembra svaporare nel nulla. La nuova rivelazione, infatti, cancella sbrigativamente l'evento sensazionale lasciando spazio a futuri pseudo-eventi. Il dolore, comunque, sembra essere l'unica certezza, l'unica verità, all'interno della comunità bergamasca: "[i]l dolore, la sofferenza: sarebbero rimasti, insepolti, soltanto quelli” (Il bambino che sognava la fine del mondo 278).

Con i suoi ritmi, la cronaca non offre spazio alla riflessione; grazie alla letteratura, invece, il lettore si sofferma su certi passaggi, li rilegge e riflette sul malessere e sulla violenza. La letteratura permette al lettore di provare empatia, aiutandolo anche a stanare il proprio dolore. La cronaca, invece, si illude, cancellando sommariamente l'evento, di eliminare anche la sofferenza vissuta dai suoi attori. Il trauma, invece, è e resta inemendabile e Antonio Scurati ce lo conferma valentemente ne Il bambino che sognava la fine del mondo.

\section{Auburn University}

\section{Opere citate}

Augé, Marc. Nonluoghi. Introduzione a una antropologia della surmodernità con una prefazione dell'autore. Tradotto da Dominique Rolland. Milano: Elèuthera, 2009.

Bartholomew, Robert. Little Green Men, Meowing Nuns and Head-Hunting Panics. Jefferson, NC: McFarland \& Company, 2001.

Baudrillard, Jean. Il delitto perfetto. La televisione ha ucciso la realtà? Tradotto da Gabriele Piana. Milano: Raffaello Cortina Editore, 1996.

. La scomparsa della realtà. Antologia di scritti. Tradotto da Augusto Zuliani. Bologna: Logo Fausto Lupetti Editore, 2009. 
. Simulacri e imposture. Bestie, Beauborg, apparenze e altri oggetti. A cura di Matteo G. Brega. Milano: Pgreco Edizioni, 2009.

. The Evil Demon of Images. Sydney: Power Institute of Fine Arts, 1987.

- The Jean Baudrillard Reader. A cura di Steve Redhead. Edinburgh:

Edinburgh University Press Ltd, 2008.

. Violenza del virtuale e realtà integrale. Tradotto da Massimo Leone. Firenze: Le Monnier, 2005.

Bauman, Zygmunt. Paura liquida. Tradotto da Marco Cupellaro. Roma: Laterza, 2012.

Berardinelli, Alfonsi, a cura di. I dieci libri dell'anno 2008/2009. Milano: 24 ORE Motta Cultura, 2009.

Boorstin, Daniel. A Guide to Pseudo-Events in America. New York: Atheneum, 1987.

Capote, Truman. In Cold Blood. New York: Vintage International Edition, 1966 [1993].

Casadei, Alberto. Stile e tradizione nel romanzo italiano contemporaneo. Bologna: il Mulino, 2007.

De Angelis, Gabriella. "Il sopravvissuto di Antonio Scurati, ovvero 'ogni memoria è falsa.'” In Hanna Serkowska, 393-406.

Di Consoli, Andrea. "Il bambino che sognava la fine del mondo di Antonio Scurati, analisi di un romanzo superstizioso." In Alfonso Berardinelli, 81-89.

Donnarumma, Raffaele. "Angosce di derealizzazione. Fiction e non-fiction nella narrativa italiana di oggi." In Hanna Serkowska, 23-50.

Donnarumma, Raffaele e Policastro, Gilda, a cura di. "Ritorno alla realtà? Otto interviste a narratori italiani." Allegoria 57 (2008): 9:25.

Falcetto, Bruno. "Ibridare finzione e realtà.” In Vittorio Spinazzola, 62-69.

Ferraris, Massimo. Manifesto del nuovo realismo. Roma-Bari: GlF editori Laterza, 2012.

Giglioli, Daniele. Senza trauma. Scrittura dell'estremo e narrativa del nuovo millennio. Macerata: Quodlibet, 2011.

Guglielmi, Angelo. "La realtà per gli scrittori." Alfabeta 2.16 (2012): 28. Web. 16 aprile 2014. http://labont.it/wordpress/wp-content/uploads/2011/11/ Guglielmi-.pdf

Lipperini, Loredana. "Scurati: basta con lo Strega. Scarpa è un buffone di corte" La Repubblica, 7 agosto 2009: 52. Web. 16 aprile 2014. http://ricerca.re- 
pubblica.it/repubblica/archivio/repubblica/2009/08/07/scurati-basta-conlo-strega-scarpa-un.html

Mazzarella, Arturo. Politiche dellirrealtà. Scritture e visioni tra Gomorra e Abu Ghraib. Torino: Bollati Boringhieri, 2011.

McLuhan, Marshall. The Medium is the Massage. Berkeley: Ginko Press, 1967.

Ming, Wu. New Italian Epic. Letteratura, sguardo obliquo, ritorno al futuro. Torino:

Einaudi, 2009. http://www.wumingfoundation.com/italiano/WM1_saggio_sul_new_italian_epic.pdf

Mondello, Elisabetta, a cura di. Roma noir 2008. "Hannibal the Cannibal c'est moi" Realismo e finzione nel romanzo noir italiano. Roma: Robin Edizioni, 2008. . "Finzione narrativa ed «effetto realtà»." In Elisabetta Mondello, 13-48.

Novelli, Mauro. "Il romanzo sociale senza morti ammazzati." In Vittorio Spinazzola, 37-42.

Pavel, Thomas. Mondi di invenzione. Realtà e immaginario narrativo. Tradotto da Andrea Carosso. Torino: Einaudi, 1986.

Sartori, Giovanni. Homo videns. Bari: Laterza, 1999.

Scurati, Antonio. Gli anni che non stiamo vivendo. Il tempo della cronaca. Milano: Bompiani, 2010.

- Il bambino che sognava la fine del mondo. Milano: Bompiani, 2009.

- Il padre infedele. Milano: Bompiani, 2013.

. Il sopravvissuto. Milano: Bompiani, 2005.

. "Intervista a Antonio Scurati." Intervista di Boris Borgato. Mangialibri.

Web. 16 aprile 2014. http://www.mangialibri.com/node/4553

. La letteratura dellinesperienza. Scrivere romanzi al tempo della televisione.

Milano: Bompiani, 2006.

. "La retorica della paura è ovunque." Intervista di Maddalena Bovenzi. Scuola di scrittura Omero. 29 marzo 2009. Web. 16 aprile 2014. http:// www.omero.it/omero-magazine/interviste/antonio-scurati-la-retorica-della-paura-e-dovunque/

- Gianni Vattimo. "Conversazione tra Antonio Scurati e Gianni Vattimo." EquiLibri. La lente di ingrandimento. Web. 27 aprile 2014. http://94.32.64.110/www.equilibrielmas.it/website/upload/archivio/X_ Conversazione_tra_Antonio_Scurati_e_Gianni_Vattimo.htm

Serkowska, Hanna, a cura di. Finzione cronaca realtà. Scambi, intrecci e prospettive nella narrativa italiana contemporanea. Massa: TransEuropa, 2011. 
Spinazzola, Vittorio, a cura di. Tirature '10. Il New Italian Realism. Milano: il Saggiatore, 2010.

Travaglio, Marco. La scomparsa dei fatti. Si prega di abolire le notizie per non disturbare le opinioni. Milano: il Saggiatore, 2006.

Žižek, Skavoj. Welcome to the Desert of the Real. Brooklyn NY: Verso, 2002. 